\title{
COVID-19 Post-Vaccinal Lymphadenopathy Mimicking Lymph Node Metastases from Breast Neoplasia
}

\section{Fabrice FOKOUE ${ }^{*}$, I. Duran DERIJCKERE ${ }^{1}$, Carlos ARTIGAS ${ }^{1}$, Erwin WOFF', Géraldine GEBHART ${ }^{1}$, Denis LARSIMONT ${ }^{2}$ and Patrick FLAMEN ${ }^{1}$}

${ }^{1}$ Department of Nuclear Medicine, Jules Bordet Institute, Free University of Brussels (ULB), Belgium

${ }^{2}$ Department of Pathology, Jules Bordet Institute, Free University of Brussels (ULB), Belgium

*Corresponding author: Dr. Fabrice FOKOUE, Department of Nuclear Medicine, Jules Bordet Institute, Free University of Brussels (ULB), Brussels, Belgium, Tel: +32 (0) 465495586

\begin{abstract}
Since the effective start of the large vaccination campaigns against the Coronavirus disease 19 (COVID-19) pandemic, case reports are rising concerning unilateral axillary lymphadenopathies highly uptaking FDG PET/CT scans. These lymphadenopathies can be a source of false positive for FDG PET/CT in a post-vaccination context. We report here the case of a 59-year-old womanfor whom a FDG PET/CT has been performed as part of the initial extension assessment of a recurrence of a left breast, 15 days after receiving her first dose of Moderna COVID 19 vaccine. Apart from the primary mammary lesion, the FDG PET CT revealed multiple unilateral axillary lymphadenopathies whose neoplastic nature was excluded after an accurate interview and confirmed after a histological examination. This case confirms the key role of a detailed interview before performing an FDG PET/CT examination to reduce false positives as much as possible.
\end{abstract}

\section{Keywords}

Covid-19 vaccination, Axillary lymphadenopathy, Breast cancer, FDG PET CT

\section{Case Report}

This is a case of a 59-year-old woman with a history of a right breast cancer in 2001 with BRCA1 mutation triple negative treated with lumpectomy, adjuvant chemotherapy and external radiotherapy. In 2019, a recurrence in the same breast is diagnosed and treated with surgery and chemotherapy.

In 2021, a new lesion is diagnosed in the upper external quadrant of the contralateral breast (triple negative, Ki67 at $80 \%$, cT1b) for which she is referred for an initial staging by FDG PET/CT [1-5].

Apart from a single moderately hypermetabolic left breast nodular lesion (SUVmax 1.7) corresponding to the neoplastic process, whole-body FDG PET/CT images showed a minimum of three moderately hypermetabolic left axillary nodes, peri and sub-centimeter size with a SUV $_{\max }$ of 2.8 for the hottest one (Figure 1).

Initially, a metastatic origin of these axillary lymph nodes could not be formally excluded given the neoplastic context. However, after an accurate interview of the patient, the first dose of Moderna ${ }^{\circledR}$ COVID-19 vaccine has been received 15 days before the FDG PET/CT with injection in the left arm, supporting an inflammatory origin.

Left breast lumpectomy with sentinel lymph node resection was performed and histopathological findings of the sentinel lymph node revealed no sign of malignancy (Figure 2).

\section{Discussion}

Nowadays time has come for widespread vaccination intending to end the pandemic. Hypermetabolic lung infiltrates have been described in patients with active COVID-19 infection. However, the influence of vaccination on FDG PET/CT is still to be investigated. Similarly to the influenza vaccine, axillary lymph nodes activation has been previously described in patients recently receiving the COVID-19 vaccine. 

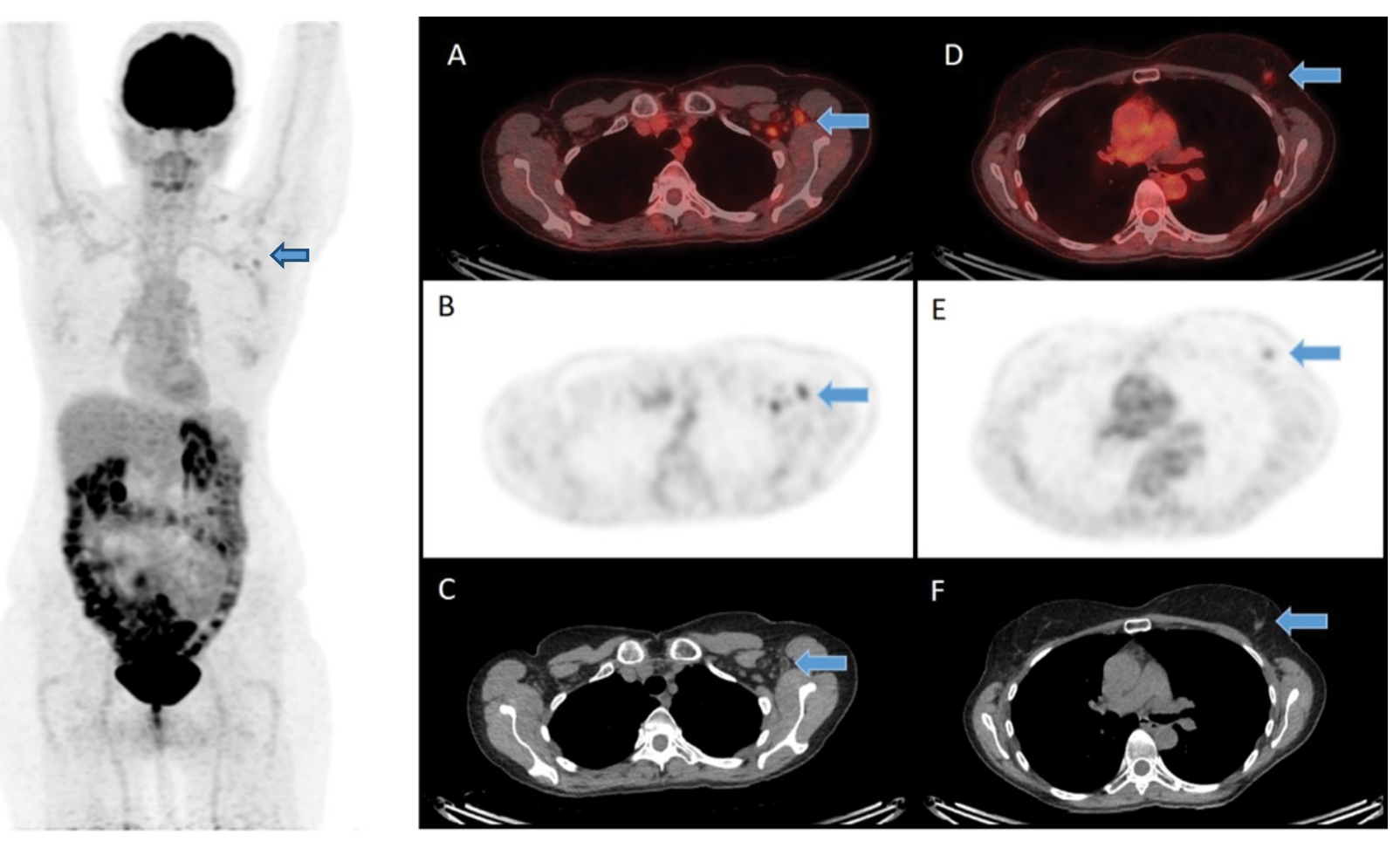

Figure 1: FDG PET images showing multiple hypermetabolic left axillary nodesat the Level II $(A, B, C)$ and level III, beside an hypermetabolic left breast nodular lesion (D, E, F) corresponding to the neoplastic process.
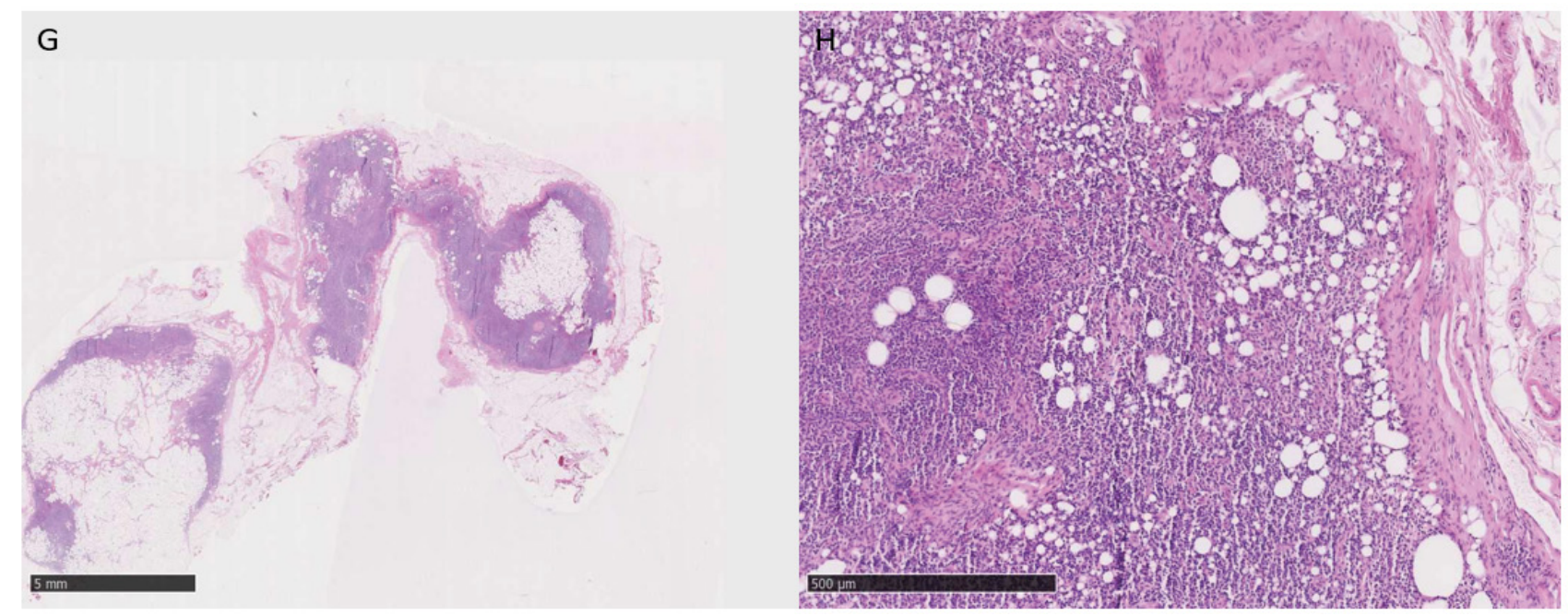

Figure 2: Hematoxylin and eosin staining under 40x magnification image shows a lymph node in adipose involution without objectified disorganization (G). 100 x magnification image shows a sinus histiocytic hyperplasia without architectural disorganization of the ganglion.

Nuclear medicine community awareness of possible pitfall findings in patients recently receiving the COVID-19 vaccine is of utmost importance to avoid misdiagnosis. Systematic interrogatory before PET/CT is highly recommended.

\section{Conclusion}

This clinical case confirms that an accurate systematic interview before PET/CT can avoid possible pitfall findings such as inflammatory lymphadenopathy reaction in patients recently receiving the COVID-19 vaccine.

\section{Patient Consent for Publication}

Obtained.

\section{Acknowledgements}

Jule Bordet Institute, Department of Nuclear Medicine.

\section{Funding}

This research did not receive any specific grant from funding agencies in the public, commercial, or not-for profit sectors. 


\section{Disclosure}

The authors report no conflicts of interest in this work.

\section{References}

1. Eifer M, Eshet $Y$ (2021) Imaging of COVID-19 vaccination at FDG PET/CT. Radiology 299.

2. Mehta N, Sales RM, Babagbemi K, Levy AD, McGrath AL, et al. (2021) Unilateral axillary adenopathy in the setting of COVID-19 vaccine. Clin Imaging 75: 12-15.
3. Nawwar AA, Searle J, Hagan I, Lyburn ID (2021) COVID-19 vaccination induced axillary nodal uptake on [18F] FDG PET/CT. Eur J Nucl Med Mol Imaging 48: 2655-2656.

4. Society of Breast Imaging (1985) SBI recommendations for managing axillary adenopathy post COVID vaccination.

5. Local Reactions, Systemic Reactions, Adverse Events, and Serious Adverse Events: Moderna COVID-19 Vaccine | CDC. 